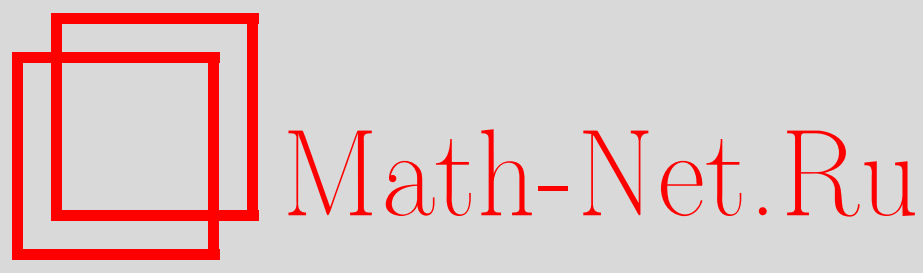

М. Н. Чернодуб, А. Вершельде, В. И. Захаров, Модель двухкомпонентной жидкости для кварк-глюонной плазмы, ТМФ, 2012, том 170, номер 2, 258-264

DOI: https://doi.org/10.4213/tmf6764

Использование Общероссийского математического портала Math-Net.Ru подразумевает, что вы прочитали и согласны с пользовательским соглашением http://www . mathnet.ru/rus/agreement

Параметры загрузки:

IP: 54.210 .77 .194

26 апреля 2023 г., 16:57:06

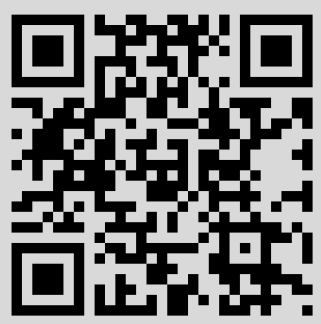




\title{
ФИЗИКА
}

Том 170, № 2

февраль, 2012

(C) 2012 г. М. Н. Чернодуб $\sigma^{* \dagger}$, А. Вершельде ${ }^{\dagger}$, В. И. Захаров

\section{МОДЕЛЬ ДВУХКОМПОНЕНТНОЙ ЖИДКОСТИ ДЛЯ КВАРК-ГЛЮОННОЙ ПЛАЗМЫ}

\begin{abstract}
Обсуждается модель двухкомпонентной жидкости для кварк-глюонной плазмы. Показано, что на качественном уровне модель объясняет естественным образом основные экспериментальные наблюдения, касающиеся свойств плазмы. С динамической точки зрения модель предполагает существование эффективного скалярного поля с отличным от нуля вакуумным ожиданием. Гипотеза о существовании такого конденсата подтверждается решеточными данными. Сформулировано, какие решеточные данные дали бы возможность проверки модели.
\end{abstract}

Ключевые слова: квантовая хромодинамика, кварк-глюонная плазма, голографические модели.

\section{1. ВВЕДЕНИЕ}

Открытие кварк-глюонной плазмы в экспериментах на ускорителе тяжелых ионов RHIC (детальное изложение этого явления, обсуждение и ссылки см., в частности, в обзорах [1]) изменило ландшафт теоретических исследований, посвященных квантовой хромодинамике (КХД). Возникла проблема объяснения экзотических свойств плазмы, обнаруженных на опыте. Новый вызов теории носит такой же фундаментальный характер, как проблема объяснения удержания кварков, и эти две проблемы взаимосвязаны. Более того, возник интерес к релятивистской гидродинамике, теории сверхтекучести и, в более общем смысле, к приложению методов голографии к изучению свойств конденсированных сред [2].

В настоящей статье мы рассматриваем возможность того, что некоторое видоизменение двухкомпонентной модели свертекучести может быть непосредственно применено к кварк-глюонной плазме. Основная идея работы та же, что и нашего сообщения [3]. Мы приводим новые аргументы в пользу применимости этой модели и обращаемся к проблеме ее проверки. В разделе 2 мы кратко суммируем основные экспериментальные сведения о свойствах плазмы и демонстрируем, что модель

*Laboratoire de Mathématiques et Physique Théorique, Université Francois-Rabelais de Tours, Tours, France. E-mail: maxim.chernodub@lmpt.univ-tours.fr

${ }^{\dagger}$ Department of Physics and Astronomy, Ghent University, Ghent, Belgium

${ }^{\ddagger}$ Институт теоретической и экспериментальной физики, Москва, Россия. E-mail: vzakharov@itep.ru 
естественным образом объясняет эти наблюдения. В разделе 3 мы даем краткий обзор решеточных данных, которые свидетельствуют в пользу существования конденсата скалярного поля. В разделе 4 мы предлагаем возможную проверку модели с помощью измерения коррелятора компонент тензора энергии-импульса.

Авторы почитают высокой для себя честью возможность представить эту статью в выпуск журнала, посвященный памяти академика Альберта Никифоровича Тавхелидзе, выдающегося физика-теоретика и организатора научных исследований, автора ряда пионерских работ по КХД, которой посвящена также и наша работа.

\section{2. КАЧЕСТВЕННЫЕ СВОЙСТВА ПЛАЗМЫ}

Представляется полезным (для построения моделей) свести все многообразие свойств плазмы к трем основным пунктам: уравнению состояния, свойству вязкости и роли квантовых эффектов.

1. Предположение о существовании плазмы было высказано давно. Более того, уравнение состояния плазмы также давно известно, поскольку оно было установлено на основе численных экспериментов в рамках решеточной формулировки КХД (ссылки см., например, в работах [1]). Оказывается, что уравнение состояния для плазмы близко́ к уравнению состояния идеального газа кварков и глюонов:

$$
[\epsilon(T)]_{\text {plasma }} \approx[\epsilon(T)]_{\text {ideal gas }}(1-\delta),
$$

где $\delta \approx 0.15$ - поправка, $\epsilon(T)$ обозначает плотность энергии как функцию температуры $T$ и $[\epsilon(T)]_{\text {ideal gas }}$ плотность энергии для невзаимодействующих кварков и глюонов. Таким образом, уравнение состояния указывает на то, что плазма близка к идеальному газу.

2. Наблюдение (1) создает иллюзию простоты свойств плазмы. Однако анализ данных, полученных на ускорителе RHIC, привел к заключению, что плазма обладает самой низкой из всех известных доныне субстанций вязкостью $\eta$ :

$$
\left(\frac{\eta}{s}\right)_{\text {plasma }} \approx \frac{1}{4 \pi},
$$

где $s$ - плотность энтропии (введенной здесь для того, чтобы представить результаты измерения вязкости в безразмерных единицах). Значение $1 / 4 \pi$ несколько символично и приводится лишь для облегчения запоминания экспериментальных данных. Истинное значение $\eta$ может быть больше, скажем $\eta / s \sim 0.4$ [1], или даже меньше [4]. Значение $\eta=1 / 4 \pi$ представляет собой гипотетический нижний предел рассматриваемого отношения [5]. Таким образом, измерения вязкости указывают на то, что плазма близка к идеальной жидкости (определенной так, что для нее $\eta=0$ ). Подчеркнем, что для идеального газа вязкость стремится к бесконечности, $\eta / s \rightarrow \infty$, точнее говоря, это отношение обратно пропорционально квадрату константы связи, $\eta / s \sim 1 / \alpha_{\mathrm{s}}^{2}$

3. В качестве вариации свойства 2 можно утверждать [5], что такое низкое значение вязкости подразумевает, что квантовые эффекты критически важны, и, строго говоря, жидкость не может рассматриваться как классическая. Действительно, используя стандартные оценки кинетической теории, легко находим, что $\eta / s \sim k_{\mathrm{B}}^{-1} \tau_{\text {relaxation }}(\epsilon / n)$, где $k_{\mathrm{B}}$ - константа Больцмана, $\tau$ - время релаксации и $n-$ 
плотность частиц. Согласно принципу неопределенности произведение энергии частицы $\epsilon / n$ на время свободного пробега $\tau_{\text {relaxation }}$ не может быть меньше постоянной Планка. Таким образом,

$$
\frac{\eta}{s} \sim \frac{\tau_{\text {relaxation }}}{\tau_{\text {quantum }}}
$$

где "квантовое время" $\tau_{\text {quantum }} \sim \hbar / k_{\mathrm{B}}$. Тогда наблюдение $(2)$ подразумевает квантовую природу кварк-глюонной плазмы.

Одновременное объяснение всех трех наблюдений (1)-(3) представляет собой вызов теории, поскольку они, на первый взгляд, противоречат друг другу. Действительно, мы начали с того, что плазма близка к идеальному газу, а заканчиваем доказательством того, что плазма представляет собой квантовую жидкость. Тем более удивительно, что оказывается относительно просто предложить модель, которая позволяет (на качественном уровне) объединить все кажущиеся противоречивыми свойства плазмы [3]. Мы имеем в виду двухкомпонентную модель свертекучести, сформулированную Ландау.

Действительно, зададимся вопросом, что “специфично” для вязкости? Возможно ли, чтобы одновременно было справедливо уравнение состояния, близкое к случаю идеального газа, и тем не менее вязкость была бы очень низкой? Представим себе, что мы имеем дело с двухкомпонентной субстанцией. Одна из компонент с весом $c_{1}$ занимает наибольший фазовый объем и ответственна за уравнение состояния. Другая компонента имеет значительно меньший весовой фактор $c_{2}$ и низкое значение вязкости $\eta_{2}$. Тогда общая вязкость жидкости может быть малой, поскольку, по крайней мере наивно, при вычислении полной вязкости складываются обратные степени парциальных вязкостей:

$$
\frac{1}{\eta_{\mathrm{tot}}}=\frac{c_{1}}{\eta_{1}}+\frac{c_{2}}{\eta_{2}},
$$

где факторы $c_{1,2}$ нормированы: $c_{1}+c_{2}=1$. Действительно, физический смысл вязкости $\eta$ подобен физическому смыслу электрического сопротивления, и если существуют два независимых движения, то применимо правило (4) для сложения параллелельных сопротивлений ${ }^{1)}$. Таким образом, двухкомпонентная модель объединяет естественным образом свойства 1 и 2, сформулированные выше. Предполагая, что одна из компонент сверхтекучая, мы получаем в качестве бонуса также объяснение свойства 3.

Уместно подчеркнуть еще одно обстоятельство. В нерелятивистском случае сверхтекучая компонента испаряется при конечной температуре $T_{\mathrm{c}}$. Легко понять физику этого явления. При $T=0$ сверхтекучая компонента связана с конденсатом частиц с импульсом $\mathbf{p}=0$, при ненулевой температуре возбуждаются состояния с ненулевым импульсом. Вследствие сохранения в нерелятивистском пределе числа частиц

1) Уравнение (4) можно найти, в частности, в старых учебниках, посвященных физике растворов [6]. На более современном языке пример сверхтекучести сам по себе может служить лучшей иллюстрацией применимости уравнения (4). Действительно, сверхтекучая фракция может быть малой и тем не менее обеспечивать сверхтекучесть всей жидкости в целом. При более детальном обсуждении этого вопроса необходимо соблюдать известную осторожность, различая вязкость по отношению к капиллярному движению и по отношению к вращениям (недавнее обсуждение этого вопроса можно найти, например, в работе [7]). 
сверхтекучая компонента исчезает при определенной температуре. В релятивистском случае, т. е. в отсутствие закона сохранения числа частиц, теоретические ограничения на фазовый объем, занимаемый сверхтекучей компонентой, слабее. Даже при $T \rightarrow \infty$ непертурбативная компонента в случае теорий Янга-Миллса обращается в ноль только логарифмически:

$$
\lim _{T \rightarrow \infty} c_{2}(T) \sim g_{\mathrm{s}}^{6}(T) \sim \frac{1}{\ln ^{3} T},
$$

где $g_{\mathrm{s}}^{2}(T)$ представляет собой константу связи в первоначальной, четырехмерной, формулировке.

\section{3. СКАЛЯРНЫЙ КОНДЕНСАТ}

3.1. Общие ограничения. С динамической точки зрения применимость сценария со сверхтекучестью зависит от существования (эффективного) скалярного поля, сконденсированного в температурном вакууме:

$$
\langle\varphi\rangle \neq 0 \text {. }
$$

Тогда фаза этого конденсата представляет собой новую легкую степень свободы. Соотношение (5) налагает очень серьезные ограничения и на более детальном уровне подразумевает следующий набор условий:

1) поле $\varphi$ комплексно: $\varphi^{*} \neq \varphi$;

2) тем не менее существование конденсата (5) не должно нарушать сохранение какого-либо квантового числа, подобного заряду;

3) в случае сверхтекучести следует скорее рассуждать в терминах трехмерного поля $\varphi(\mathbf{r})$, поскольку производная поля по времени фиксирована, $\partial_{t} \varphi=\mu$, где $\mu-$ химический потенциал, сопряженный заряду, которым обладает поле $\varphi$ (обобщение последнего равенства на случай релятивистской плазмы можно найти в работе [8]).

3.2. Термический скаляр. Условия 1-3, сформулированные в предыдущем пункте, будучи рассмотренными абстрактно, представляются очень трудными для выполнения. Тем более знаменательно, что трехмерное поле с похожими свойствами естественно возникает [9] в струнной модели фазового перехода к деконфайнменту и обычно называется термическим скаляром (прекрасный обзор и дальнейшее обсуждение можно найти в работе [10]).

Рассмотрим температуру ниже и близкую к температуре фазового перехода $T_{\mathrm{c}}$. В струнной картине $\beta_{\mathrm{c}} \equiv 1 / T_{\mathrm{c}}=1 / \alpha^{\prime}$ где $2 \pi \alpha^{\prime} \equiv l_{\mathrm{s}}^{-2}$ есть натяжение струны. При $T=T_{\text {c }}$ статистическая сумма по всем струнным состояниям начинает расходиться. Основное утверждение состоит в том, что при малых значениях $\left|T-T_{\mathrm{c}}\right|$ вся сумма насыщается вкладом одной степени свободы, а именно скалярным мезоном с массой

$$
m_{\beta}^{2} \approx \frac{\beta_{\mathrm{c}}\left(\beta_{\mathrm{c}}-\beta\right)}{2 \pi^{2}\left(\alpha^{\prime}\right)^{2}}
$$

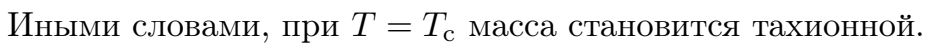

Для более детального анализа удобно воспользоваться полимерным представлением теории поля скалярной частицы, согласно которой действие, ассоциированное 
с траекторией длины $L$, равно $S=M L$, где $M$ обозначает затравочную массу частицы. Траектории представляют собой случайные блуждания частицы с перенормированной массой. Соответственно, свободная энергия термического скаляра может быть представлена как сумма по случайным блужданиям:

$$
F=\beta \ln Z=\beta \int_{0}^{\infty} \frac{d L}{L} \frac{e^{-m_{\beta}^{2} l_{\mathrm{s}} L}}{\left(l_{\mathrm{s}} L\right)^{d / 2}},
$$

где $d$ - число пространственных координат, в нашем случае $d=3$. Выражение $(6)$ достаточно характерно для полимерного подхода, его специфической чертой является то, что $l_{\mathrm{s}}$ играет роль длины ребра решетки и выражается через натяжение струны.

Основное утверждение состоит в том, что свободная энергия термического скаляра явным образом совпадает со статистической суммой одной статичной струны с натяжением $1 / 2 \pi \alpha^{\prime}$. Более того, одна длинная струна доминирует в статистической сумме для газа струн.

3.3. Трехмерный скаляр при $T>T_{\mathrm{c}}$. Вопрос о том, что происходит с термическим скаляром при $T>T_{\text {c }}$, остается открытым; например, может происходить конденсация скалярного поля, такой сценарий, в частности, характерен для теории перколяции. Основные аспекты этого явления можно понять из соотношения (6). При $m_{\beta}^{2}=0$ экспоненциальное подавление при больших длинах $L$ пропадает, однако интеграл по длинам $L$ все еще расходится в ультрафиолетовом, но не в ин-

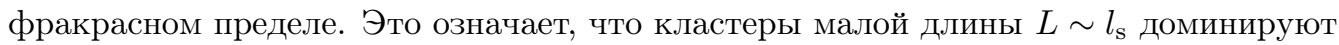
в сумме. Вероятность наблюдения больших длин пропорциональна обратной степени длины $L$ и подавлена численно при $L \rightarrow \infty$. В случае тахионной массы скаляра возникает кластер бесконечной длины, но плотность кластера пропорциональна положительной степени фактора $\left|m_{\beta}^{2}\right|$ и подавлена численно при температурах выше и близких к $T_{\mathrm{c}}$. На языке теории поля возникновение бесконечного кластера соответствует ненулевому конденсату, $\langle\phi\rangle \neq 0$.

Представим себе, что термический скаляр на самом деле конденсируется при $T>T_{\text {c }}$. Тогда, что весьма примечательно, условия, которые мы сформулировали выше, оказываются выполненными. Действительно, имеет место следующее:

1) термический скаляр описывается комплексным полем, поскольку интегрирование в соотношении (6) идет по замкнутым петлям, что соответствует комплексному полю на языке полимерного представления теории поля;

2) термический скаляр ассоциирован с топологическим квантовым числом, котоpoе есть не что иное, как число закруток вокруг временно́й координаты, компактифицированной при конечной температуре в евклидовом пространстве;

3) термический скаляр представляет собой трехмерное скалярное поле, как это следует из представления (6).

В настоящее время общепринято рассматривать дуальные модели теорий Янга-Миллса, которые представляют собой струнные модели в пространствах с дополнительными измерениями. Появление термического скаляра при температурах

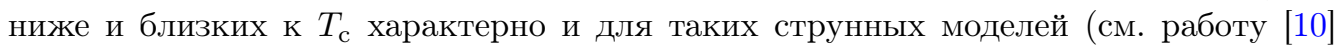
и ссылки в ней). Нельзя, однако, утверждать, что наивная версия конденсации термического скаляра реализуется в этих дуальных моделях, скорее фазовый переход 
представляет собой изменение геометрии в дополнительных измерениях. Скаляр-

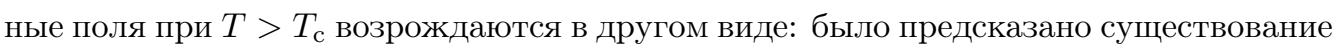

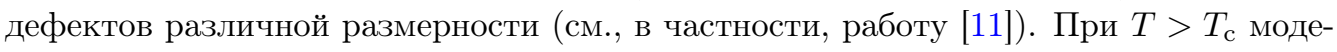
ли предсказывают существование струн, ориентированных по временно́й оси. Тогда их трехмерные проекции выглядят как траектории и действительно соответствуют трехмерным скалярным частицам. Существуют независимые решеточные данные, которые, по всей видимости, подтверждают эти предсказания теории [12].

Подводя итог, отметим, что имеются убедительные свидетельства в пользу су-

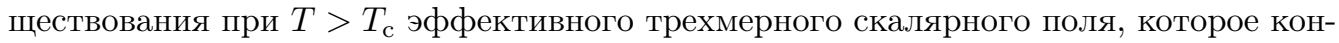
денсируется в термическом вакууме КХД. Существование такого скалярного поля представляет собой необходимое условие справедливости двухкомпонентной модели.

\section{4. ВОЗМОЖНАЯ ПРОВЕРКА МОДЕЛИ}

Соображения, приведенные выше, демонстрируют, что двухкомпонентная модель кварк-глюонной плазмы не противоречит существующим данным. Нельзя, однако, утверждать, что модель действительно подтверждена этими данными. Проверка модели может быть осуществлена с помощью решеточных измерений коррелятора компонент тензора энергии-импульса $T^{0 i}, i=1,2,3$. Более подробно, рассмотрим запаздывающую функцию Грина

$$
G_{\mathrm{R}}^{0 j, 0 i}(k) \equiv i \int d^{4} x e^{-i k x} \theta(t)\left\langle\left[T^{0 j}(x), T^{0 i}(0)\right]\right\rangle .
$$

Сконцентрируемся на случае нулевой частоты, $k_{0}=0$. Существуют два независимых формфактора, которые отвечают поперечным и продольным волнам:

$$
G_{\mathrm{R}}^{0 j, 0 i}(0, \mathbf{k})=\frac{k^{i} k^{j}}{\mathbf{k}^{2}} G_{\mathrm{R}}^{\mathrm{L}}(\mathbf{k})+\left(\delta^{i j}-\frac{k^{i} k^{j}}{\mathbf{k}^{2}}\right) G_{\mathrm{R}}^{\mathrm{T}}(\mathbf{k}) .
$$

Вклад свертекучей компоненты в $G_{\mathrm{R}}^{\mathrm{L}, \mathrm{T}}$ обсуждался во многих статьях и учебниках. Здесь мы приведем результат, полученный в работе [13], где учтены также релятивистские поправки:

$$
\lim _{\mathbf{k} \rightarrow 0} G_{\mathrm{R}}^{\mathrm{T}}(\mathbf{k})=-\left(s T+\mu \rho_{\mathrm{n}}\right), \quad \lim _{\mathbf{k} \rightarrow 0} G_{\mathrm{R}}^{\mathrm{L}}(\mathbf{k})=-(s T+\mu \rho),
$$

где $\rho \equiv \rho_{\mathrm{n}}+\rho_{\mathrm{s}}$ есть полная плотность, в то время как $\rho_{\mathrm{n}}$ и $\rho_{\mathrm{s}}$ суть плотности нормальной и сверхтекучей компонент соответственно. Иными словами,

$$
\lim _{\mathbf{k} \rightarrow 0} G_{\mathrm{R}}^{0 j, 0 i}(0, \mathbf{k})=\text { const } \cdot \frac{k^{i} k^{j}}{\mathbf{k}^{2}}+\text { контактные члены. }
$$

Здесь константа пропорциональна плотности сверхтекучей компоненты $\rho_{\mathrm{s}}$, и только существование сверхтекучей компоненты приводит к неаналитичности при малых $\mathbf{k}$. Это утверждение представляет собой общее свойство всех моделей сверхтекучести, и было бы важным попытаться обнаружить неаналитичность в решеточных измерениях.

Отметим, что предложенная проверка модели (см. соотношение (10)) относится к статическим величинам, мы положили $k_{0}=0$. Поскольку нет зависимости от частоты, продолжение евклидова пространства в пространство Минковского носит непосредственный характер, и предсказание модели $\rho_{\mathrm{s}} \neq 0$ может быть проверено в решеточных измерениях. 


\section{5. ЗАКЛЮЧЕНИЕ}

Представляется замечательным, что известные качественные свойства кваркглюонной плазмы, по-видимому, указывают на применимость двухкомпонентной модели сверхтекучести. В терминах теории поля эта модель подразумевает конденсацию эффективного трехмерного скалярного поля, что предположительно можно подтвердить решеточными данными. Проверку модели можно провести путем поиска неаналитичности в корреляторе компонент тензора энергии-импульса на решетке.

\section{Список литературы}

[1] E. V. Shuryak, Nucl. Phys. A, 750:1 (2005), 64-83, arXiv: hep-ph/0405066; Th. Schäfer, D. Teaney, Rept. Prog. Phys., 72:12 (2009), 126001, 40 pp., arXiv: 0904.3107; D. Teaney, Prog. Part. Nucl. Phys., 62:2 (2009), 451-461.

[2] S. A. Hartnoll, Class. Quantum Grav., 26:22 (2009), 224002, 61 pp., arXiv: 0903.3246.

[3] M. N. Chernodub, H. Verschelde, V.I. Zakharov, Nucl. Phys. B Proc. Suppl., 207-208 (2010), 325-328, arXiv: 0905.2520.

[4] M. Luzum, P. Romatschke, Phys. Rev. C, 78:3 (2008), 034915, 17 pp.; Erratum, 79:3 (2009), 039903, 4 pp., arXiv: 0804.4015.

[5] P. Kovtun, D. T. Son, A. O. Starinets, Phys. Rev. Lett., 94:11 (2005), 111601, 4 pp., arXiv: hep-th/0405231.

[6] Ch. H. Lees, Phil. Mag., 6:1 (1901), 128-147; L. A. Utracki, Polymer Blends Handbook, Springer, New York, 2003.

[7] S. Koh, The onset of superfluidity in capillary flow of liquid helium 4, arXiv: 0808.4030.

[8] D. T. Son, Internat. J. Modern Phys. A., 16S1C (2001), 1284-1286, arXiv: hep-ph/0011246.

[9] J. J. Atick, E. Witten, Nucl. Phys. B, 310:2 (1988), 291-334; B. Sathiapalan, Phys. Rev. D, 35:10 (1987), 3277-3279; Я. И. Коган, Писвма в ЖКЭТФ, 45:12 (1987), 556-559.

[10] M. Kruczenski, A. Lawrence, JHEP, 07 (2006), 031, 29 pp., arXiv: hep-th/0508148.

[11] A. S. Gorsky, V. I. Zakharov, A. R. Zhitnitsky, Phys. Rev. D, 79:10 (2009), 106003, 9 pp., arXiv: 0902.1842.

[12] M. N. Chernodub, A. Nakamura, V.I. Zakharov, Deconfinement phase transition in mirror of symmetries, arXiv: 0904.0946.

[13] Ch. P. Herzog, A. Yarom, Phys. Rev. D, 80:10 (2009), 106002, 13 pp., arXiv: 0906.4810. 